a. Consultora, Hospital Nacional de Pediatría J. P. Garrahan, Ciudad Autónoma de Buenos Aires.

b. Sección Hepatología Infantil, Hospital Municipal de Niños de San Justo, Provincia de Buenos Aires.

c. Hepatología y Trasplante Hepático Pediátrico, Hospital Alemán, Ciudad Autónoma de Buenos Aires.

d. Sección Nutrición Infantil, Hospital Universitario Austral, Pilar, Provincia de Buenos Aires.

e. Hepatología, Hospital de Pediatría J. P. Garrahan, Ciudad Autónoma de Buenos Aires.

f. Hospital de Día Polivalente, Hospital de Niños Ricardo Gutiérrez, Ciudad Autónoma de Buenos Aires.

g. Sección Hígado, Vías Biliares y Páncreas, Hospital Nacional Prof. Alejandro Posadas, El Palomar, Provincia de Buenos Aires.

h. Unidad 4, Hepatología, Hospital de Niños Ricardo Gutiérrez, Ciudad Autónoma de Buenos Aires.

i. Hepatología y Trasplante Hepático, Unidad de Hígado, Hospital Universitario Austral, Pilar, Provincia de Buenos Aires.

j. Instituto de Estudios Inmunológicos y Fisiopatológicos (IIFP), Departamento de Ciencias Biológicas, Facultad de Ciencias Exactas, Universidad Nacional de La Plata y Consejo Nacional de Investigaciones Científicas y Técnicas (CONICET), La Plata, Provincia de Buenos Aires.

Correspondencia: Dra. Luisa Bay, bay.luisa@gmail.com

Financiamiento:

Ninguno.

Conflicto de intereses: los autores Dra. Luisa Bay, Dr. Federico Piñero, Dr. Eduardo Fassio y Dra. Paula Rozenfeld declaran recibir honorarios por disertaciones de carácter docente del laboratorio Alexion.

Recibido: 22-8-2016 Aceptado: 28-11-2016

\title{
Enfermedad hepática y dislipemia como manifestación de deficiencia de lipasa ácida lisosomal (LAL-D). Aspectos clínicos, diagnósticos y nuevo tratamiento. Actualización
}

Liver disease and dyslipidemia as a manifestation of lysosomal acid lipase deficiency (LAL-D). Clinical and diagnostic aspects, and a new treatment. An update

\author{
Dra. Luisa Bay ${ }^{a}$, Dra. Cristina Cañero Velasco ${ }^{b}$, Dra. Mirta Cioccac ${ }^{c}$ Dra. Andrea Cotti ${ }^{d}$, \\ Dra. Miriam Cuarterolo ${ }^{e}$, Dr. Alejandro Fainboimf, Dr. Eduardo Fassiog, \\ Dra. Marcela Galoppo ${ }^{h}$, Dr. Federico Piñero ${ }^{i}$ y Dra. Paula Rozenfeld
}

\section{RESUMEN}

La deficiencia de lipasa ácida lisosomal es una enfermedad genética aún poco reconocida, con significativa morbimortalidad en niños y en adultos.

Esta guía orienta sobre cuándo sospechar la enfermedad y cómodiagnosticarla.Serecomienda agregar la deficiencia de lipasa ácida lisosomal a la lista de diagnósticos diferenciales de las sepsis, enfermedades oncológicas, enfermedades de depósito, diarrea prolongada y desnutrición crónica y linfohistiocitosis hemofagocítica. Asimismo, se sugiere considerarla en pacientes jóvenes con dislipemia y arterioesclerosis y en enfermedades que ocurran con hígado graso y/o hepatomegalia.

La hepatomegalia, hiperlipidemia y / o elevación de las transaminasas en ocasión de controles de rutina o de otras afecciones deberían hacer sospechar la deficiencia de lipasa ácida lisosomal, aligual que en pacientes con cirrosis criptogénica. Hoy existe la opción de un tratamiento de remplazo enzimático específico.

Palabras clave: cirrosis, dislipidemias, enfermedad de Wolman, esteatosis hepática no alcohólica, deficiencia de lipasa ácida lisosomal.

http:/ / dx.doi.org/10.5546/ aap.2017.287

Texto completo en inglés:

http:/ / dx.doi.org/10.5546/ aap.2017.eng.287

Cómo citar: Bay L, Cañero Velasco C, Ciocca M, et al. Enfermedad hepática y dislipemia como manifestación de deficiencia de lipasa ácida lisosomal (LAL-D). Aspectos clínicos, diagnósticos y nuevo tratamiento. Actualización. Arch Argent Pediatr 2017;115(3):287-293.

\section{INTRODUCCIÓN}

La enfermedad hepática y la dislipidemia son hallazgos frecuentes en la práctica diaria y signos comunes en la deficiencia de la enzima lipasa ácida lisosomal (LAL-D), una enfermedad de depósito, de baja prevalencia. Su herencia es autosómica recesiva, causada por mutaciones en el gen LIPA, localizado en el cromosoma 10q23.2-23.3. ${ }^{1}$ Esta enzima interviene en el metabolismo y degradación de los ésteres de colesterol y triglicéridos, que se acumulan en células de diferentes tejidos si la enzima está disminuida o ausente. Los más afectados son el hígado, el bazo, las glándulas suprarrenales, el sistema hematopoyético, el endotelio vascular, el intestino y los ganglios linfáticos. Las manifestaciones clínicas de la LAL-D varían con la edad. ${ }^{2} \mathrm{El}$ compromiso hepático y la dislipidemia dominan el cuadro clínico, por lo que algunos pacientes pueden ser erróneamente diagnosticados.

\section{OBJETIVO.}

Realizar una revisión de la enfermedad, sus manifestaciones clínicas, los diagnósticos diferenciales, la confirmación diagnóstica y las novedades terapéuticas.

\section{Manifestaciones clínicas}

La edad de presentación, el espectro clínico y la progresión de la enfermedad son ampliamente variables. Hay una forma grave del lactante o enfermedad de Wolman ${ }^{4,5}$, descrita en 1956, y una forma tardía del niño, adolescente o adulto, conocida como enfermedad de depósito de ésteres de colesterol (cholesteryl ester storage disease; CESD, por sus siglas en inglés), descrita en 
1963. ${ }^{2}$ El fenotipo depende del tipo de mutación del gen LIPA y de la magnitud de la deficiencia enzimática.

La forma grave se presenta en los primeros meses de vida, con una actividad enzimática (LAL) casi ausente o $<1 \%$ de la normal. ${ }^{5}$ Los niños impresionan sanos al nacer $\mathrm{y}$, entre el primer y el segundo mes, presentan mal estado general, falla de crecimiento, desnutrición progresiva, marcada distensión abdominal por malabsorción y hepatoesplenomegalia, vómitos recurrentes, diarrea crónica (esteatorrea) y compromiso hepático con rápido desarrollo de fibrosis y / o colestasis y / o insuficiencia hepática. Se observa un aumento de las transaminasas (en el 50\%-95\% de los casos) e hiperesplenismo (anemia/plaquetopenia). ${ }^{2}$ Alrededor del $50 \%$ de los pacientes tiene calcificaciones de las glándulas suprarrenales, con la particularidad de que son bilaterales. ${ }^{6,7}$

El colesterol es elevado o normal con disminución de lipoproteínas de alta densidad (high-density lipoprotein cholesterol; HDL-C, por sus siglas en inglés) y aumento de las lipoproteínas de muy baja densidad (very low-density lipoprotein; VLDL, por sus siglas en inglés); los triglicéridos pueden estar normales o elevados. ${ }^{8}$ La presencia de retardo madurativo y/o irritabilidad es secundaria a la desnutrición, al compromiso general y a la hospitalización, ya que la enfermedad no compromete el sistema nervioso central. ${ }^{9}$ La evolución natural de esta forma clínica lleva a la muerte en el primer año de vida. ${ }^{10-12}$

El subtipo que comienza en la infancia, la adolescencia o la adultez tiene 1\%-12\% de la actividad enzimática normal; la acumulación de lípidos lleva a un daño hepático progresivo, con elevación de transaminasas, esteatosis, fibrosis y / o cirrosis. Tienen dislipemia crónica, que conduce a una arterioesclerosis temprana (infarto, aneurismas, accidente cerebrovascular). ${ }^{13-14}$

En una serie de 48 pacientes de este subtipo, la edad mediana de reporte de la enfermedad fue 5,8 años, con un máximo de 46 ; el 56\% tenía menos de 20 años y el 17\%, menos de 10. Los síntomas comenzaron antes de los 10 años en el 75\% de los pacientes. Las elevaciones de alanina aminotransferasa (ALT), aspartato aminotransferasa (AST) y gamma-glutamil transpeptidasa (GGT) se observaron en el 92\%, $59 \%$ y $20 \%$ de los casos, respectivamente. La hepatomegalia, menos frecuente que en la forma temprana, fue reportada en el $54 \%$ de los pacientes y la esplenomegalia, solo en un $23 \% .{ }^{16}$
La dislipidemia es indistinguible de la de otras etiologías más comunes. Hay aumento de la concentración de colesterol total, LDL-C, VLDL-C y triglicéridos, y disminución de los niveles de HDL-C. ${ }^{1,45}$ En pacientes pediátricos, se observan concentraciones de LDL $\geq 130 \mathrm{mg}$ / dl y HDL $\leq 45 \mathrm{mg} / \mathrm{dl} .{ }^{11}$ Se ha descrito el infarto cerebral con hiperlipemia a los 13 años y disfunción vesicular sintomática. ${ }^{16}$ (Tabla 1 ).

\section{Prevalencia}

Si bien se han descrito más de 40 mutaciones, ${ }^{17}$ la mutación en el exón 8, E8SJM (c.894G > A), se identificó en el 50\%-60\% de los pacientes con la forma de presentación tardía y ancestros europeos. ${ }^{18}$ En individuos alemanes sanos, se la detectó con una frecuencia de, aproximadamente, 1:200 de heterocigotas. Así, se puede esperar una prevalencia de 1:40 000 recién nacidos alemanes y de 1:300 000 sujetos de raza caucásica e hispanos. ${ }^{19,20}$ El número de casos reportados, mucho más bajo que estas estimaciones, sugiere que habría subdiagnóstico, probablemente, por lo inespecífico del cuadro clínico y la ausencia de un fenotipo característico. No hay estudios de prevalencia de LAL-D en la Argentina.

\section{Diagnóstico de la deficiencia de lipasa ácida lisosomal en el laboratorio}

El diagnóstico definitivo de LAL-D se establece al demostrar la deficiencia o la ausencia de la actividad de la enzima LAL (EC 3.1.1.13) en leucocitos aislados de sangre periférica o cultivo de fibroblastos. ${ }^{21}$ La determinación enzimática puede también realizarse en gotas de sangre seca en papel de filtro, ${ }^{22}$ lo que facilita el envío de muestras al laboratorio de referencia desde lugares remotos, la realización del diagnóstico retrospectivo y el tamizaje a nivel poblacional. No obstante, si en las muestras en papel de filtro se obtiene un resultado anormal, este se debe confirmar en leucocitos o fibroblastos. Este ensayo es de utilidad para el diagnóstico confirmatorio de homocigotas, pero no así para heterocigotas, quienes pueden presentar una actividad enzimática normal.

El estudio genético molecular consiste en identificar las mutaciones patogénicas en ambos alelos del gen LIPA del paciente y se considera un ensayo diagnóstico complementario. ${ }^{23} \mathrm{La}$ identificación de las mutaciones resulta de utilidad para el estudio familiar y para poder, así, ofrecer diagnóstico a los heterocigotas de la familia y asesoramiento genético. 
TABLa 1. Fenotipo de deficiencia de lipasa ácida lisosomal: de forma grave del lactante (Wolman) a forma de inicio tardío del niño, adolescente y adulto (enfermedad por depósito de ésteres del colesterol) (modificada de Grawoski, 2014)

\begin{tabular}{lcc}
\hline Signos clínicos & Recién nacido y lactante & Niños, adolescentes y adultos \\
\hline Edad de presentación & $0-3$ meses & De la infancia a la adultez \\
Edad de fallecimiento & \pm 6 meses & Variable \\
Hepatomegalia & ++++ & $+/++$ \\
Fibrosis hepática/ cirrosis & ++ & $+/+++$ \\
Esplenomegalia & +++ & $+/-$ \\
Malabsorción & +++ & $+/-$ \\
Caquexia & +++ & \pm \\
Calcificaciones adrenales & ++ & $++/+++$ \\
Dislipemia & \pm & $150-350$ veces lo normal \\
Depósito de ésteres de colesterol & $5-160$ veces lo normal & $1,5-3$ veces lo normal \\
Depósito de triglicéridos & En el hígado: 2-10 veces lo normal \\
& En el bazo: 8-100 veces lo normal & \\
\end{tabular}

Figura 1. A. Efecto de la deficiencia de LAL en el hígado y metabolismo lipídico. El colesterol LDL y VLDL es captado por el receptor del hepatocito y conducido al lisosoma. La deficiencia de LAL lleva a la acumulación de ésteres de colesterol (EC) y triglicéridos, y reduce la producción de colesterol libre (CL) y ácidos grasos ( AG). La reducción del colesterol aumenta la actividad de sterol regulatory-element binding protein (SREBPS) y aumenta la sintesis de colesterol y $A G$, con aumento de colesterol $L D L$ y VLDL. La reducción de CL reduce la activación de los receptores X hepáticos (LXR) y la expresión de transportador ABCA1, lo que disminuye el eflujo de colesterol a la apolipoproteína A1 (ApoA-1) y reduce el colesterol HDL plasmático. B. Efecto del tratamiento con sebelipasa alfa (rhLAL); captada por el receptor manosa-6-fosfato (M6P) es llevada al lisosoma donde revierte los defectos en el metabolismo lipídico. Evita la acumulación de lípidos en el hepatocito. Modificado de Rader D. ${ }^{24}$

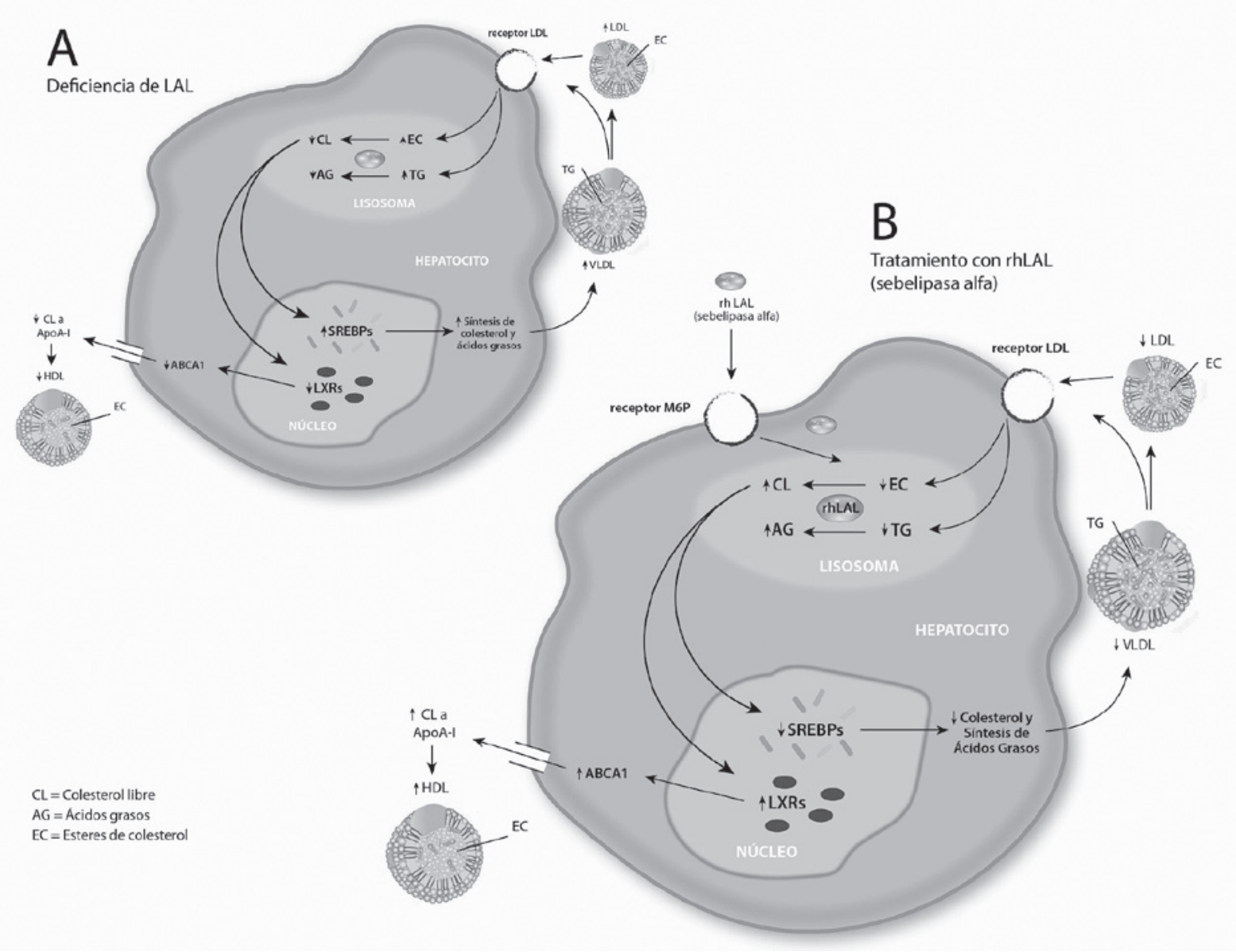

LAL: lipasa ácida lisosomal; HDL: lipoproteínas de alta densidad; LDL: lipoproteínas de baja densidad;

VLDL: lipoproteínas de muy baja densidad; TG: triglicéridos. 


\section{Fisiopatología}

La lipasa ácida actúa sobre el metabolismo lipídico en el hepatocito y su deficiencia, tal como se describe fisiopatológicamente en la Figura 1. A, explica la dislipidemia y la acumulación de lípidos neutros en el lisosoma, que lleva al daño hepático. ${ }^{24}$

\section{Diagnóstico diferencial}

Aunque existen pruebas bioquímicas y genéticas de diagnóstico de LAL-D, el reconocimiento de la enfermedad depende de la sospecha clínica, que es baja. ${ }^{11,12}$ Las manifestaciones clínicas y bioquímicas se comparten con otras enfermedades cardiovasculares, hepáticas o metabólicas.,12 Debe incorporarse al listado de diagnósticos diferenciales de enfermedades hepáticas y de dislipidemias. Dadas su morbilidad grave y mortalidad prematura, conviene que los pacientes sean diagnosticados con rapidez, antes de que se desarrolle daño permanente de los órganos (hígado, bazo, sistema digestivo, sistema vascular). ${ }^{11,12}$ Para ello, revisaremos los diagnósticos diferenciales.

La visceromegalia en la forma grave del lactante o enfermedad de Wolman se comparte con otras enfermedades de depósito (Niemann-Pick, Gaucher, glucogenosis IV). Con linfohistiocitosis hemofagocítica, se debe tener en cuenta que se ha descrito coincidencia de esta con LAL-D ${ }^{25}$ y otras enfermedades oncológicas. Si bien estas otras entidades tienen rasgos fenotípicos y bioquímicos propios, el cuadro clínico grave, del primer año de vida, puede confundirse con la deficiencia de lipasa ácida. ${ }^{26-31}$

Las calcificaciones suprarrenales deberán diferenciarse del neuroblastoma y de la hemorragia suprarrenal, aunque, en estos casos, la lesión suele ser unilateral.

Sepsis, infecciones congénitas con visceromegalia, gastroenteritis subaguda o crónica, cuadros de malabsorción, enfermedad de Hirschsprung y alergia a la leche de vaca pueden confundirse con LAL-D, ${ }^{21}$ por lo que se incluirá entre los diagnósticos diferenciales cuando alguna de las etiologías más frecuentes no se confirme o la evolución del paciente no sea la esperada.

En pacientes mayores (niño, adolescente y adulto), con peso normal o sobrepeso, colesterol total y LDL-C elevados, HDL-C bajo, aumento de transaminasas y/o hepatomegalia y/o esteatosis hepática, se debe incluir LAL-D entre los diagnósticos diferenciales. ${ }^{12}$

En pacientes con sobrepeso u obesidad, con síndrome metabólico, ${ }^{32}$ transaminasas elevadas, signos de esteatosis hepática en los estudios por imágenes y una ingesta de etanol $<140 \mathrm{~g} /$ semana, además de hígado graso no alcohólico (nonalcoholic fatty liver disease; NAFLD, por sus siglas en inglés) / esteatohepatitis no alcohólica (nonalcoholic steatohepatitis; NASH, por sus siglas en inglés), es necesario descartar otras enfermedades musculares o hepáticas. ${ }^{32,33}$

La obesidad per se no justifica hacer el diagnóstico de NAFLD por transaminasas elevadas $^{34}$ y se recomienda excluir la LAL-D en aquellos pacientes que, al cumplir el tratamiento y bajar de peso, no modifican los datos bioquímicos según lo esperado. 25,35

TABLA 2. Diagnóstico diferencial de hígado graso no alcohólico/esteatohepatitis no alcohólica y deficiencia de lipasa ácida lisosomal (forma tardía)

\begin{tabular}{lcc}
\hline & NAFLD/NASH & LAL-D (tardía) \\
\hline Prevalencia & $20 / 100$ & $1: 40000$ \\
Hepatomegalia & $+/-$ & +++ \\
Esplenomegalia & $+/-$ & ++ \\
IMC & Sobrepeso/obesidad & Normal/sobrepeso \\
AST / ALT $\uparrow$ & $20 \%$ en población general & +++ \\
Metabolismo de glúcidos & Anormal & Normal \\
Triglicéridos & $\uparrow \uparrow$ & Normal o \\
HDL-C & $\downarrow$ & $\downarrow$ \\
LDL-C & Normal o $\uparrow$ & $\uparrow$ \\
Biopsia hepática & Esteatosis macrovesicular & Esteatosis microvesicular \\
\hline
\end{tabular}

NAFLD/NASH: hígado graso no alcohólico/esteatohepatitis no alcohólica; IMC: índice de masa corporal; ALT: alanina aminotransferasa; AST: aspartato aminotransferasa; HDL-C: lipoproteínas de alta densidad; LDL-C: lipoproteínas de baja densidad. 
Al no haber un marcador bioquímico específico, para NAFLD y NASH, se ha sugerido realizar una biopsia hepática, ${ }^{36,37}$ aunque tampoco da un diagnóstico de certeza. Los pacientes con esteatosis microvacuolar pueden ser erróneamente diagnosticados como NAFLD ${ }^{35} \mathrm{O}$ como NASH. El NAFLD es, actualmente, la causa más frecuente del aumento de las transaminasas en la niñez y adolescencia, atribuido al incremento de la incidencia de obesidad. ${ }^{38,39}$ En el NAFLD en adolescentes y adultos, por lo general, hay hiperglucemia con resistencia a la insulina y no así en la LAL-D. ${ }^{40}$ El NAFLD es excepcional en menores de 3 años e infrecuente en menores de 10. Las características de NAFLD-NASH y LAL-D se ilustran en la Tabla 2.

La cirrosis ${ }^{41}$ en América Latina se asocia a alcoholismo crónico, virus de hepatitis (virus de hepatitis C-VHC-: 29\%; virus de hepatitis B -VHB-: 27\%), a alteraciones del drenaje hepático $(19 \%)$, a NASH $(8 \%)$ y a cirrosis criptogénica $(6 \%){ }^{42}$

En pediatría, de $5 \%$ a $15 \%$ de los casos de cirrosis son criptogénicos $y$, tanto en niños como en adultos, debe incluirse la investigación de LAL-D.

La esteatosis hepática microvacuolar o mixta en niños y adolescentes lleva a investigar la enfermedad de Wilson, en la que la esteatosis es secundaria a la acumulación de cobre, con más frecuencia que a pensar en LAL-D, aunque esta última debe también incluirse entre los diagnósticos diferenciales. ${ }^{43,35}$

Con insuficiencia hepática temprana, además de investigar tirosinemia, galactosemia, defectos de $\beta$ oxidación, hemocromatosis neonatal y enfermedades mitocondriales, debemos incluir LAL-D. ${ }^{44,45}$

En LAL-D, la histología hepática muestra acumulación de ésteres de colesterol en hepatocitos y células de Kupffer. Casi el 50\% presenta fibrosis septal con progresión a cirrosis. Puede requerirse un trasplante hepático dentro de los tres años siguientes al inicio de los síntomas. ${ }^{6,11,12} \mathrm{El}$ diagnóstico histológico está facilitado por la inmunotinción de la proteína lisosomal catepsina D. ${ }^{11}$

En cuanto a la dislipemia, en la infancia, hay recomendaciones nacionales para su tamizaje desde los 6 a los 17 años. ${ }^{46,47}$ Así, se detectan casos de hipercolesterolemia plausible de tratamiento temprano. Cuando no hay antecedentes familiares claros y no hay mejoría con el tratamiento habitual (dieta y actividad física para la normalización del índice de masa corporal -IMC-), se sugiere investigar la LAL-D, así como cuando se descartan causas de hipercolesterolemia secundaria.

Los pacientes pediátricos con LDL $>130 \mathrm{mg} / \mathrm{dl}$ y/o HDL $<40 \mathrm{mg} / \mathrm{dl}$ sin antecedentes familiares claros, sin obesidad ni distribución central del tejido adiposo con glucemia normal en ayunas podrían padecer LAL-D ${ }^{48}$ (Tabla 3$) .{ }^{8}$

\section{TRATAMIENTO}

No ha habido tratamientos efectivos para la LAL-D; los hipolipemiantes han sido poco efectivos y no mejoran el compromiso hepático. ${ }^{1,11}$ El trasplante hepático tiene pocos antecedentes y escaso seguimiento, aunque, en el pasado, ha sido la única alternativa posible en casos con evolución a insuficiencia hepática terminal. ${ }^{1}$

TABLA 3. Poblaciones de alto riesgo por considerar para el cribado para la deficiencia de lipasa ácida lisosomal

Modificado de Burton B, JPGN 2015;61:619-625. ${ }^{8}$

Pacientes no obesos con lo siguiente:

- Hepatomegalia persistente

- Transaminasas altas sin causa definida

- LDL alto (> $130 \mathrm{mg} / \mathrm{dl}, 4,1 \mathrm{mmol} / \mathrm{L}$ )

- HDL bajo (varones $<40 \mathrm{mg} / \mathrm{dl}$; mujeres $<50 \mathrm{mg} / \mathrm{dl}$ )

Pacientes obesos con diagnóstico de síndrome metabólico y lo siguiente:

- Hepatomegalia persistente

- Ausencia de hiperglucemia

- Transaminasas elevadas sin causa definida, que no mejoran al disminuir su IMC

Pacientes obesos que disminuyen su IMC pero que no mejora su lipidograma Pacientes con esteatosis microvesicular

HDL: lipoproteínas de alta densidad; LDL: lipoproteínas de baja densidad; IMC: índice de masa corporal. 
En 2015, fue aprobado el uso de una enzima recombinante humana de remplazo (sebelipasa) por vía endovenosa, cada 15 días. En un estudio doble ciego ${ }^{49}$ multicéntrico, aleatorizado, se demostró una disminución significativa en las transaminasas, del contenido de grasa hepática medida con resonancia magnética nuclear (RMN) y de la hepatomegalia. El LDL-C y los triglicéridos disminuyeron significativamente y aumentó el HDL-C. Los efectos adversos en el estudio fueron pocos y leves (Figura 1. B).

En un ensayo fase II/III con sebelipasa alfa, en niños con la forma grave de comienzo temprano, se mostró que 5 de 9 sobrevivieron más allá de los 2 años y uno, 5 años. Mejoraron marcadores de enfermedad hepática, síntomas gastrointestinales y el estado nutricional..$^{50}$

Si bien aún no hay seguimiento a largo plazo con este tratamiento, sebelipasa, por su mecanismo de acción, tendría un potencial efecto en reducir la fibrosis hepática y su progresión a la cirrosis, así como en mejorar la dislipemia y evitar sus complicaciones cardiovasculares. ${ }^{51}$

\section{REFERENCIAS}

1. Reiner Z, Guardamagna O, Nair D, Soran H, et al. Lysosomal acid lipase deficiency an under-recognized cause of dyslipidaemia and liver dysfunction. Atherosclerosis 2014;235(1):21-30.

2. Grabowski G, Charnas L, Du H. Lysosomal Acid Lipase Deficiencies: The Wolman Disease/Cholesteryl Ester Storage Disease Spectrum. In Valle D, Beaudet AL, Vogelstein B, eds. The Online Metabolic and Molecular Bases of inherited Diseases. New York: McGraw-Hill, 2013.

3. Konno T, FujiiM, WatanukiT,KoizumiK.Wolman's disease: the first case in Japan. Tohoku J Exp Med 1966;90(4):375-89.

4. Crocker AC, Vawter GF, Neuhauser EB, Rosowsky A. Wolman's disease: three new patients with a recently described lipidosis. Pediatrics 1965;35:627-40.

5. Gómez-Nájera M, Barajas-Medina H, Gallegos-Rivas M, Mendez-Sashida P, et al. New Diagnostic Method for Lysosomal Acid Lipase Deficiency and the Need to recognize its Manifestation in infants (Wolman Disease). J Pediatr Gastroenterol Nutr 2015;60(3):e22-4.

6. Abramov A, Schorr S, Wolman M. Generalized xanthomatosis with calcified adrenals. AMA J Dis Child 1956;91(3):282-6.

7. Amitai N, Grozovski S, Landau D. Adrenal calcification in an infant with cholestasis. J Pediatr 2015;167(5):1163.

8. Burton BK, Deegan PB, Enns GM, Guardamagna O, et al. Clinical Features of Lysosomal Acid Lipase Deficiency. J Pediatr Gastroenterol Nutr 2015;61(6):619-25.

9. Bernstein DL, Hülkova H, Bialer MG, DenickRJ. Cholesteryl ester storage disease: review of the findings in 135 reported patients with an underdiagnosed disease. $J$ Hepatol 2013;58(6):1230-43.

10. Anderson RA, Bryson GM, Parks JS. Lysosomal acid lipase mutations that determine phenotype in Wolman and cholesterol ester storage disease. Mol Genet Metab 1999;68(3):333-45.
11. Marshall WC, Ockenden BG, Fosbrooke AS, Cumings JN. Wolman's disease. A rare lipidosis with adrenal calcification. Arch Dis Child 1969;44(235):331-41.

12. Mayatepek E, Seedorf U, Wiebusch H, Lenhartz H, et al. Fatal genetic defect causing Wolman disease. J Inherit Metab Dis 1999;22(1):93-4.

13. Sjouke B, Van der Stappen JW, Groener JE, Pepping A, et al. Hypercholesterolaemia and hepatosplenomegaly: Two manifestations of cholesteryl ester storage disease. Neth J Med 2015;73(3):129-32.

14. Decarlis S, Agostoni C, FerranteF, ScarlinoS, etal.Combined hyperlipidaemia as a presenting sign of cholesteryl ester storage disease. J Inherit Metab Dis 2009;32(Suppl 1):S11-3.

15. Dalgıç B, Sarı S, Gündüz M, Ezgü F, et al. Cholesteryl ester storage disease in a young child presenting as isolated hepatomegaly treated with simvastatin. Turk J Pediatr 2006;48(2):148-51.

16. Haller W, Sharif K, Millar A, Brown R, et al. Gallbladder Dysfunction in Cholesterol Ester Storage Disease. J Pediatr Gastroenterol Nutr 2010;50(5):555-8.

17. Stenson PD, Mort M, Ball EV, Howells K, et al. The Human gene mutation database: 2008 update. Genome Med 2009;22(1):13.

18. Klima H, Aslanidis $C$, Fehringer $\mathrm{P}$, Lackner KJ, et al. A splice junction mutation causes deletion of a 72-base exón from the mRNA for lysosomal acid lipase in a patient with cholesteryl ester storage disease. J Clin Invest 1993;92(6):2713-8.

19. Scott SA, Liu B, Nazarenko I, Martis S, et al. Frequency of the cholesteryl ester storage disease common LIPA E8SJM mutation (c.894G $>$ A) in various racial and ethnic groups. Hepatology 2013;58(3):958-65.

20. Muntoni S, Wiebusch H, Jansen-Rust M, Rust S, et al. Prevalence of cholesteryl ester storage disease. Arterioscler Thromb Vasc Biol 2007;27(8):1866-8.

21. Guy GJ, Butterworth J. Acid esterase activity in cultured skin fibroblasts and amniotic fluid cells using 4-methylumbelliferyl palmitate. Clin Chim Acta 1978;84(3):361-71.

22. Hamilton J, Jones I, Srivastava R, Galloway P. New method for the measurement of lysosomal acid lipase in dried blood spots using the inhibitor Lalistat 2. Clin Chim Acta 2012;413(15-16):1207-10.

23. Fasano T, Pisciotta L, Bocchi L, Guardamagna O, et al. Lysosomal lipase deficiency: molecular characterization of eleven patients with Wolman or cholesteryl ester storage disease. Mol Genet Metab 2012;105(3):450-6.

24. RaderD. Lysosomal Acid Lipase Deficiency-A new therapy for a genetic Lipid disease. N Engl J Med 2015; 373(11): 1071-3.

25. Rabah F, Al-Hashmi N, Beshlawi I. Wolman's Disease with secondary Hemophagocytic Lymphohistiocytosis. Pediatr Hematol Oncol 2014;31(6):576-8.

26. HoffmanEP, Barr ML, Giovanni MA, Murray M. Lysosomal Acid Lipase Deficiency. In Pagon RA, Adam MP, Ardinger $\mathrm{HH}$, et al, eds. GeneReviews ${ }^{\circledR}$ [Internet]. Seattle (WA): University of Washington; 2016. [Access: November 30, 2016]. Available from: http://www.ncbi.nlm.nih.gov/ books/NBK305870/.

27. Moses SW, Parvari R. The variable presentation of glycogen storage disease type IV: a review of clinical enzymatic and molecular studies. Curr Mol Med 2002;2(2):178-88.

28. Mignot C, Gelot A, Bessières B, Daffos F, et al. Perinatallethal Gaucher disease. Am JMed Genet A 2003;120A(3):33844.

29. Grabowsky GA, Andria G, Baldellou A, et al. Pediatric non-neuropathic Gaucher disease: presentation, diagnosis and assessment. Consensus statment. Eur J Pediatr 2004;163(2):58-66. 
30. Vanier MT, Millat G. Niemann Pick disease type C. Clin Genet 2003;64(4):269-81.

31. Niemann-Pick disease. En Nyhan W, Barshop B, Al-Apeel A. Atlas of Inherited Metabolic Diseases. 3rd ed. London: Hodder Arnold; 2012:708-17.

32. Grundy SM, Brewer HB Jr, Cleeman JI, Smith SC Jr, et al. Definition of metabolic syndrome: Report of the National Heart, Lung, and Blood Institute/American Heart Association conference on scientific issues related to definition. Circulation 2004;109(3):433-8.

33 WGO Global Guidelines. Non-alcoholic fatty liver disease and nonalcoholic steatohepatits. 2012. [Acceso: 30 de noviembre de 2016]. Disponible en: http:/ / www. worldgastroenterology.org/UserFiles / file/guidelines / nafld-nash-english-2012.pdf.

34. Vajro P, Lenta S, Socha P, Dhawan A, et al. Diagnosis of nonalcoholic fatty liver disease in children and adolescent: position paper of the ESPGHAN. Hepatology Committee. J Pediatr Gastroenterol Nutr 2012;54(5):700-13.

35. Chalasani N, Younossi Z, Lavine JE, Diehi AM, et al. The diagnosis and management of non-alcoholic fatty liver disease: Practice Guideline by the American Association for the Study of Liver Diseases, American College of Gastroenterology, and the American Gastroenterological Association.. Hepatology 2012;55(6):2005-23.

36. Neuschwander-Tetri BA, Caldwell SH. Nonalcoholic steatohepatitis: Summary of an AASLD Single Topic Conference. Hepatology 2003;37(5):1202-19.

37. Brunt EM, Kleiner DE, Wilson LA, Belt $\mathrm{P}$, et al. Nonalcoholic fatty liver disease (NAFLD) activity score and the histopathologic diagnosis in NAFLD: distinct clinicopathologic meanings. Hepatology 2011;53(3):810-20.

38. Santos LF, Hernández G, Varón Puerta A, Beltrán O, et al. Enfermedad hepática por infiltración grasa no alcohólica. La nueva pandemia del milenio. Rev Col Gastroenterol 2010;25(4):380-98.

39. WHO. Report of the commission on ending childhood obesity. Ginebra: WHO; 2016. [Acceso: 30 de noviembre de 2016]. Disponible en: http://apps.who.int/iris/ bitstream/10665/204176/1/9789241510066_eng.pdf.

40. Semple RK, Sleigh A, Murgatroyd PR, Adams CA, et al. Postreceptor insulin resistance contributes to human dyslipidemia and hepatic steatosis. J Clin Invest 2009;119(2):315-22.

41. McCormick PA. Hepatic Cirrhosis. En: Dooley JA, Lok ASF, Burton AK, Heathcote EJ, eds. Sherlock's Diseases of the Liver and Biliary System. 12th ed. Oxford: WilleyBlackwell;2011:103-20.

42. Piñero F, Tisi Baña M, de Ataide EC, Hoyos Duque S, et al. Liver Transplantation for Hepatocellular carcinoma: Evaluation of the alfa-fetoprotein model in a multicenter cohort from Latin America. Liver Int 2016;36(11):1657-67.

43. Rodríguez-Castro KI, Hevia-Urrutia FJ, Sturniolo GC. Wilson's disease: A review of what we have learned. World J Hepatol 2015;7(29):2859-70.

44. Molleston JP, Sokol RJ, Karnsakul W, Miethke A et al. Evaluation of the Child With Suspected Mitochondrial Liver Disease. J Pediatr Gastroenterol Nutr 2013;57(3):269-76.

45. Bliksrud Y, Ellingsen A, Bjørås M. Fumarylacetoacetate inhibits the initial step of the base excision repair pathway: implication for the pathogenesis of tyrosinemia type I. J Inherit Metab Dis 2013;36(5):773-8.

46. Expert Panel on Integrated Guidelines for Cardiovascular Health and Risk Reduction in Children and Adolescents: Summary Report. Pediatrics 2011;128(Suppl 5):S213-56.

47. Comité De Nutrición. Consenso sobre Manejo de las Dislipidemias en Pediatría. Arch Argent Pediatr 2015;113(2):177-86.

48. Alberti K, Zimmet P, Shaw J. Metabolic syndrome-a new world-wide definition. A Consensus Statement from the International Diabetes Federation. Diabet Med 2006;23(5):469-80.

49. Burton BK, Balwani M, FeilletF, Barić I, et al. A Phase 3 Trial of Sebelipase Alfa in Lysosomal Acid Lipase Deficiency. N Engl J Med 2015;373(11):1010-20.

50. Valayannopoulos V, Plantaz D, Vara R. Eckert S, et al. Clinical effect of sebelipase alfa on survival and growth in infants with lysosomal acid lipase deficiency (Wolmam Disease). Mol Genet Metab 2014,111(2):S108.

51. Paton DM. Sebelipase alfa: enzymatic replacement treatment for lysosomal acid lipase deficiency. Drugs Today (Barc) 2016:52(5):287-93. 